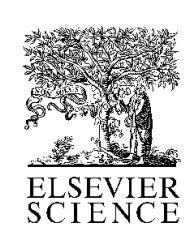

\title{
Adding a Lens Improves Spinning Speed Characterization
}

\author{
Eugene Mihaliuk and Terry Gullion* \\ West Virginia University, Morgantown WV 26506
}

Elsevier use only: Received date here; revised date here; accepted date here

\begin{abstract}
Highly stable sample rotation is important in many solid-state NMR experiments. Whether the necessary stability is achieved is not always clear. Typically only an average frequency over some time interval (often relatively long and unknown) is available from the spinning speed controller readout, which is not representative of the short-term variations of instantaneous rotation frequency. The necessity of the relatively slow measurement of spinning speed is a consequence of phase noise in the tachometer, which prevents speed measurement to be both rapid and precise at the same time. We show that adding a lens to the tachometer, without any other changes in the probe, reduces phase noise by nearly an order of magnitude and allows improved measurement of the spinning speed.
\end{abstract}

(C) 2015 Elsevier Science. All rights reserved.

MAS, spinning speed control

\section{Introduction}

Magic-angle sample spinning (MAS) NMR has become routine, especially since Schaefer and Stejskal combined MAS with cross-polarization [1-3]. Commercial probes and spinning speed controllers make sample spinning easy, and at moderate speeds MAS requires no more attention than properly setting the stator angle and dialing in the desired speed into the spinning speed controller. However, some rotor synchronous pulse sequences, including specialized variations of REDOR [4-6], demand extremely precise synchronization between sample rotation and RF pulse trains, and without good spinning speed control the observed signal intensity can be greatly compromised $[7,8]$. In these cases it is particularly important to understand the details of measurement and control of sample spinning.

Although theory and practice of frequency and phase measurement are extremely well developed in electrical engineering and metrology $[9,10]$, the topic is seldom discussed systematically in the NMR context. The stability of spinning speed is most often reported in the literature as a range within which the readout of the spinning speed controller remained while the experimenter cared to monitor it, as this is usually the only data available to the user. This is analogous to specifying the frequency of a peak in an NMR spectrum without saying anything about its width or shape. Controllers do not provide much information about the quality of sample spinning to the user. Usually the only information that is provided is the average spinning speed that is determined over a relatively long measurement interval. Although the dynamics of feedback loops in the controller is typically faster than the display update rate, neither is specified and even controllers with a faster feedback loop do not display the short-term deviations of the spinning speed. This makes comparison between reported spinning speeds rather less meaningful, while the actual dynamics of instantaneous rotation frequency remains unknown.

Evidently, experiments for which standard stability of the spinning speed is not sufficient remain, as indicated by suggestions for improving sample spinning stability $[11,12]$. At the same time, even when lack of spinning

*Corresponding author. Fax: +1-304-293-4906
E-mail address: terry.gullion@mail.wvu.edu 
speed stability is suspected of causing problems, rigorous analysis of spinning speed stability is hardly ever attempted. Due to lack of diagnostic data on the instantaneous spinning speed, it is generally presumed and hoped, but seldom known with certainty, that any changes in the speed are sufficiently slow to be unimportant for a particular experiment.

The instantaneous rotation frequency can be determined as a reciprocal of the rotation period, and it can be measured, in principle, for each revolution. Unfortunately, this is impossible to achieve in practice using current NMR hardware. A typical MAS probe tachometer reliably produces a pulse per rotor revolution, sufficient for the majority of MAS NMR experiments. However, from turn to turn the tachometer pulses correspond to slightly different angular orientations of the rotor. While of little importance when the rotational frequency is measured as the number of pulses per second, this effect becomes crippling when spinning speed estimates are attempted by accurately timing a small number of revolutions.

When synchronization of elements of the pulse sequence to the tachometer signal is employed [13], the jitter in tachometer pulse timing is also a concern. In principle, the synchronization between sample spinning and the NMR pulse programmer reduces the problem of accurately maintaining the spinning speed for rotor-synchronous pulse sequences by preventing accumulation of phase error across multiple rotor periods despite small deviations of rotation frequency from its set point. However, the jitter (phase noise) in the tachometer signal limits the accuracy with which angular orientation of the rotating sample is known. This fact has prompted development of various techniques to "clean" the tachometer output and reduce the amount of phase noise. These methods often include averaging tachometer phase by using a phase locked loop [14-16] or implementation of Kalman filtering techniques $[17,18]$ to optimally combine several sources of information and produce the best possible estimate of the rotation frequency under existing measurement noise and uncertain dynamics of the system [19].

Here, we investigate the root cause of the problem of why the tachometer output does not accurately represent angular position of the spinning rotor. Then we show that adding a focusing lens to the tachometer, without any additional changes to the probe, provides a simple way to reduce the phase noise by nearly an order of magnitude. To be clear, we note that this modification by itself will not improve spinning speed stability but what it will do is provide a means to more accurately determine rotational periods over a short time frame.

\section{Probe Tachometer}

Typical solid-state MAS probes contain an optical tachometer to measure the rotation frequency. It illuminates the sample rotor and measures the intensity of light scattered back by a small area of the rotor, on which one or more regions are painted or darkened by laser etching. During the course of rotation of the rotor this mark passes through the view field of the tachometer and thus modulates the intensity of backscattered light collected by the tachometer. The optical system is usually trivially simple and consists of two optical fibers, one for illumination and one for detection, with their ends a short distance away and facing the rotor (style A), or a single, larger, bifurcated optical fiber bundle which both illuminates and collects back scattered light (style B). In both cases, the backscattered light is then converted to an electrical signal by a photodiode. A threshold crossing detection circuit then converts the amplified photocurrent into a train of digital pulses corresponding to changes in intensity of the backscattered light due to rotation of the rotor. These pulses are the output signal of the tachometer, and they are used by the electronics in the spinning speed controller to monitor and control sample rotation.

For each revolution, the start of the electric pulse ideally coincides with specific orientation of the sample rotor. In practice, noise in the tachometer electronics makes the timing of the pulse to deviate slightly from the ideal position as illustrated in Figure 1. The noise $e_{n}$ causing the uncertainty in the time of threshold crossing $\Delta t$ is exacerbated by the relatively slow transition between the "dark" and "bright" conditions caused by a relatively large size of the area from which the back scattered light is collected. As the blackened mark on the rotor enters the illuminated area, the backscattered light intensity gradually diminishes with rotation of the rotor and then returns to its previous high intensity level as the blackened sector leaves the illuminated area. The magnitude of the jitter $\Delta \mathrm{t}$ is equal to the magnitude of the amplifier noise $\mathrm{e}_{\mathrm{n}}$ divided by the slope of the signal edge. If the observed dark-bright transition could be made more rapid, the time variation would be reduced correspondingly.

\section{Phase Noise and Jitter}

NMR practitioners are familiar with the Fourier transform and the connection that it provides between the representation of a signal as a function of time and a representation of the same signal in terms of spectral density as a function of frequency. Deviation of any physical process from ideal periodicity appears in both domains; it is reflected as the repetitions of the signal being non-identical from one time to the next and as a spectral line shape having finite width [9, 20]. For nearly periodic processes, the short-term non-ideal behavior is described as phase noise (as opposed to arbitrarily longer term random or systematic frequency drift). The phase noise corresponds to "jitter" in the time domain, where jitter is commonly viewed as the signal transitions deviating from 
the expected positions. In the frequency domain the same phase noise is described as signal power density at frequencies slightly offset from the mean value of fundamental frequency thereby giving rise to the spectral line shape. An adequate description of the phase noise requires either the specification of the jitter as a function of time delay or the power density as a function of frequency offset.

Magic-angle sample spinning is an approximately periodic process and, as such, is only partially specified by its mean frequency. A more accurate description of the sample rotation requires knowledge of the properties of its phase noise and frequency drift. Although it is in principle possible to monitor the "line shape" of the sample spinning and frequency drift using a high-resolution audio-frequency spectrum analyzer [21], a more simple and convenient diagnostic procedure is to directly observe the tachometer signal on an oscilloscope. The oscilloscope trace is triggered by the rising (or falling) edge of the tachometer signal crossing the trigger threshold, which should be set at a fixed level at the steepest part of the edge. By design of the oscilloscope, the position of the trigger point and the signal trace in its vicinity will appear stationary on the screen. Portions of the same signal that are further away from the trigger point will however not retrace each other on successive repetitions, generally diverging further apart at longer time delays if the process that generates the signal exhibits random phase and frequency variations. (However, we note that if the frequency undergoes periodic changes of any kind, no matter how large the amplitude of the changes is, the average over any integral number of periods would remain the same as long as all the periods are identical. For example, if the drive pressure oscillates at $30 \mathrm{~Hz}$, then the rotational frequency measured over any multiple of $33.3 \mathrm{~ms}$ will remain constant, despite large periodic variations of instantaneous frequency.) Many oscilloscopes have a useful feature that allows observing the signal at a considerable delay from the trigger point, regardless of the currently set horizontal scale; this feature is the delayed time base on older analog oscilloscopes in the "run after delay" mode and the simple horizontal display offset on many newer digital models. For example, observing the signal at a $10 \mathrm{~ms}$ delay from the trigger point with 1us/div resolution leads to any variation of frequency to be magnified such that a change by $100 \mathrm{ppm}$ results in a shift of horizontal position of the trace by one division of the reticle.

By observing the signal at a gradually increasing time delay between the trigger point and the observation region, one can study the jitter and frequency variation as a function of time on a time scale relevant for NMR pulse trains. If the oscilloscope allows the digital representation of the waveform of the signal to be saved, then the jitter as a function of delay and its effects on the pulse sequence can be calculated.
At short delays, the measured jitter appears to be essentially independent of the delay, and it is dominated not by a random walk in the NMR relevant physical phase of the rotor, but by the aforementioned electrical noise in the tachometer electro-optical pick-up. Only on the longer time scale does the gradual increase in the measured jitter start to reflect the underlying dynamics of the physical rotation. (If the tachometer jitter represented actual variation in the mechanical phase of the rotor, we would expect it to accumulate incrementally, as a random walk and grow as a square root of time, assuming white phase noise. Frequency drift would result in phase deviation growing with time even faster.)

Our cursory examination of several commercial and home-made probes, with rotors from 3.2 to $14 \mathrm{~mm}$ diameter, have shown that they all exhibit a slight random variation in the timing of individual tachometer pulses, often on the order of $1 \%$ of the rotor period. This jitter is annoying for several reasons and will be discussed in detail in the following sections.

For the described above method of observing jitter to be useful in debugging spinning speed problems on NMRrelevant time scale, the intrinsic tachometer jitter needs to be substantially reduced. In short, if we estimate instantaneous rotor frequency by measuring with high resolution the duration of a single rotor revolution, $1 \%$ jitter results in $+/-50 \mathrm{~Hz}$ of spurious variation at $5 \mathrm{KHz}$ spinning. If the frequency is measured over multiple periods, the variation becomes proportionally smaller for simple measurement techniques. At a relatively long measurement interval such as 1 second, the influence of this source of noise becomes completely unnoticeable and is the reason many NMR users are not aware of its existence.

\section{Frequency Measurement}

Jitter in the tachometer signal (measurement noise) and the actual mechanical fluctuations of the rotation frequency (uncertainty in the process dynamics) together set a definite theoretical limit to the accuracy with which rotation frequency can be estimated and controlled from the tachometer signal. If the rotation frequency were perfectly constant, any amount of signal jitter could be averaged out to any desired accuracy by choosing a sufficiently long averaging time, thereby producing an arbitrarily accurate estimate of frequency.

As the real rotation frequency fluctuates, the measurement time needs to be kept sufficiently short for the measurements to represent the actual, varying instantaneous rotation frequency. The more rapidly the frequency changes, the greater the deviation between the real and the estimated instantaneous frequency is for the same measurement interval. A compromise must be made between the desire to average jitter out by making the measurement interval longer and the desire to reduce the 
difference between the real and the estimated instantaneous frequency by making the measurement interval shorter. Optimal estimation theory prescribes the optimal in the least squares sense measurement procedure based on the amount of noise in the measuring system, the amount of noise in dynamics of the measured system, and the parameters of the system dynamics [17]. Since our system is very simple, satisfactory results are obtained by choosing the measurement interval that makes the two factors contribute approximately equally to the uncertainty of the measurement. The choice of the best averaging time must be determined using the measured parameters of the sample spinning process.

\section{Experimental}

A typical probe tachometer operates on diffuse backscattered light. By attaching a small lens to the stator, with no other changes to the probe, we have reconfigured the tachometer to operate with a focused light beam that essentially converts the optical system from diffused light to imaging mode. This principle is illustrated in Figure 2. Specifically, we have modified the tachometer in a Bruker $4 \mathrm{~mm}$ MAS probe by attaching a molded polycarbonate lens to the stator such as to focus the tachometer light beam on the surface of the rotor containing the speed detection mark. The stator with the lens is shown in Figure 3. The Bruker probe uses a fiber optic bundle, with half the fibers used for illumination and half used to capture reflected light. Hence, the lens serves to focus the illuminating light onto the rotor and to collimate the reflected light onto the fiber optic bundle (see Figure 2). We used an objective lens salvaged from a surplus HD/DVD-ROM module (model number Toshiba PHR803T, purchased on-line for $\$ 9$ at newegg.com). The lens is plano-convex, with a molded rim of outside diameter $5 \mathrm{~mm}$ and a $4 \mathrm{~mm}$ optical aperture; the focal length is approximately $3.5 \mathrm{~mm}$. Although the aspherical lens is optimized specifically for HD/DVDROM application, it appears to perform quite well for our needs and is close to an ideal size and focal length to function without any additional changes to the probe.

Addition of the lens allows the tachometer to pick up light from a much smaller area of the rotor compared to the original configuration. Therefore, movement of the darkened sector produces much more rapid changes in the detected light intensity. More rapid transitions directly translate into smaller variation in the timing of the tachometer output with respect to the mechanical orientation of the rotor, as shown in Figure 1, and discussed in the following section.

To rapidly measure instantaneous rotation frequency, we have interfaced the tachometer to a microcontroller (Microchip dsPIC30F2010) which allows us to measure the absolute time of the falling edge of every tachometer pulse with 50 ns resolution. The data were transmitted in real time to a PC over a 1.25 Mbps serial link, and the data were processed and visualized with a set of simple Matlab (Mathworks) scripts.

\section{Results}

A comparison between the unmodified and focused tachometer signals is shown in Figure 4. Comparison of jitter in the tachometer signal with and without lens, is shown as histograms in panels (a) and (b). The histogram of the phase distribution shows a $\mathrm{x} 5$ improvement in the phase noise after the addition of the lens. Corresponding rise and fall times of the optical signal with and without a lens are illustrated in Figure 1, which explain the observed improvement in tachometer jitter. We further observe that:

- Frequency averaged over 1 second may remain substantially constant despite large instantaneous frequency deviations.

- The assumption that the rotational frequency does not change rapidly is generally not true even for relatively large rotors $(4-5 \mathrm{~mm})$ and is likely to be even less valid for smaller, rapidly spinning rotors, making precise synchronization of RF pulses with sample rotation difficult to achieve without rapidly acting speed control.

- Due to the limitations of the typical optical arrangement, the tachometer output in most MAS probes exhibits jitter on the order of a few mechanical degrees or rotor orientation. This affects performance of rotorsynchronous pulse sequences. It also makes it difficult to rapidly monitor dynamics of sample rotation.

- Adding a lens to the tachometer optics reduces jitter by an order of magnitude and allows proportionally faster and/or more accurate monitoring of sample rotation.

- Improved measurements allow faster feedback in the MAS controller and correspondingly tighter control of angular frequency and rotor phase. Faster feedback is especially desirable when sudden disturbances are common, such as in sample reorientation in SAS probes [22-25].

The last bulleted note hints at a way to improve spinning speed stability. No matter how quickly the spinning speed is determined, there is a lag in time to make corrections to the spinning speed. For example, the air supply hoses connecting the probe to the controller are typically five to ten feet long and the control mechanism is a mechanical valve. Hence, once a correction signal for spinning speed adjustment is sent to the valve it still takes time for the change in drive pressure adjustment to be effective at the rotor. We 
recently reported a way to control the spinning speed using a heating mechanism located directly on the stator, and this arrangement makes changes to the spinning speed very rapidly because of its close proximity to the rotor (effectively eliminating time lags associated with long air hoses and mechanical valves) [26]. A combination of using a lens to better measure the spinning speed over a very short measurement window with a heating element to make necessary fast corrections in spinning speed suggests a way to better control the spinning speed.

\section{Conclusions}

We have added a small lens to the tachometer in a Bruker MAS NMR probe. The lens focuses the light in a tighter spot and reduces uncertainty in the timing of tachometer pulses by an order of magnitude, which allows us to measure rotation frequency of the sample more rapidly and more accurately. Since spinning speed can only be controlled as precisely as it can be measured, the improvement has direct impact on achievable stability of MAS speed and the synchronization between sample rotation and the elements of NMR pulse sequences.

For routine experiments, improved tachometer performance may perhaps be only useful as an aid in diagnosing spinning problems. For example, the magnitude of rapid variations of instantaneous frequency can serve as an objective diagnostic parameter similar to a more subjective "pure tone" quality of the sound of a well spinning rotor [21].

On the other hand, reduction in the tachometer phase noise can be directly useful for experiments sensitive to precision of sample spinning to RF synchronization and achieving the necessary synchronization without using complex tachometer signal filtering methods.

Lower phase noise also directly translates into possibility of measuring and controlling speed more rapidly, which may be necessary for dynamic reorientation of the spinning sample, as in SAS [22-25].

We recommend adding a lens to the tachometer optics as a simple means to achieve more accurate synchronization between sample rotation and RF pulses. The combination of using heat to control the spinning speed and addition of the lens may offer a way to more tightly control the spinning speed.

\section{Acknowledgement}

This work was supported by Grant CHE-1152009 from the National Science Foundation.

\section{Figure Captions}

Figure 1. Jitter in the tachometer output. The top of the sketch schematically shows the timing mark on the rotor crossing the indicated view field of the tachometer. Below it is shown the corresponding change in the backscattered light intensity as a function of time. At the bottom is the corresponding digital output of the tachometer. Panel (b) shows the effect of reducing the tachometer view field with respect to that in panel (a). For a given amount of electrical and optical noise $\left(\mathrm{e}_{\mathrm{n}}\right)$ making the point of threshold crossing uncertain, the corresponding jitter $\Delta \mathrm{t}$ is inversely proportional to the slope of the light intensity signal as a function of time.

Figure 2. Sketch of the light path with (b) and without a lens (a).

Figure 3. Focusing lens added to the $4 \mathrm{~mm}$ Bruker stator. Lens aperture is $4 \mathrm{~mm}$ in diameter, $\mathrm{F}=3.5 \mathrm{~mm}$. The lens was simply attached flush to the original stator housing (i.e., the stator was used as is).

Figure 4. Period of rotation (c), measured without lens ("Standard") and with the lens ("With Lens"). The "Standard" data set was taken with an average spinning speed of $1200 \mathrm{~Hz}$, and the "With Lens" data set was taken with an average spinning speed of $1198 \mathrm{~Hz}$. These two data sets were purposely taken with slightly different spinning speeds to make a more visually appealing comparison. Panels (a) and (b) show corresponding histograms of period variation. 


\section{References}

1. I.J. Lowe, Free induction decays of rotating solids, Phys. Rev. Lett 2 (1959) 285-287.

2. E.R. Andrew, A. Bradbury, R.G. Eades, Removal of dipolar broadening of nuclear magnetic resonance spectra of solids by specimen rotation, Nature 183 (1959) 18021803.

3. J.Schaefer, E.O.Strejskal, Carbon-13 nuclear magnetic resonance of polymers spinning at the magic angle, J. Am. Chem. Soc 98 (1976) 1031-1032.

4. T. Gullion and J. Schaefer, Rotational-echo doubleresonance NMR, J. Magn. Reson. 81 (1989) 196-200.

5. T. Gullion and J. Schaefer, Detection of weak heteronuclear dipolar coupling by rotational-echo doubleresonance nuclear magnetic resonance, Advances in Magnetic Resonance, W. S. Warren, Ed., Vol. 13, Academic Press, San Diego (1989) 57-83.

6. T. Gullion, Introduction to rotational-echo, doubleresonance NMR, Concepts in Magn. Reson. 10 (1998) 277289.

7. Matthias Ernst, Arno P. M. Kentgens, and Beat H. Meier, 2D exchange NMR spectra under slow MAS: A simplified scheme to obtain pure-phase spectra without unwanted cross peaks, J. Magn. Reson. 138 (1999) 66-73.

8. J.R. Garbow, T. Gullion, The importance of precise timing in pulsed, rotor-synchronous MAS NMR, Chem. Phys. Lett. 192 (1992) 71-76.

9. David W. Allan, Time and Frequency (Time-Domain) Characterization, Estimation, and Prediction of Precision Clocks and Oscillators, IEEE Trans. on Ultasonics, Ferroelectrics, and Freq. Control 34 (1987) 647-654 (Also published as NIST TN-127).

10. Fundamentals of the Electronic Counters, Application Note 200 Electronic Counter Series, Hewlett Packard Company 1997.

11. H.J. Jakobsen, A.R. Hove, H. Bildsøe, J. Skibsted, M. Brorson, Long-term stability of rotor-controlled MAS frequencies to $0.1 \mathrm{~Hz}$ proved by 14N MAS NMR experiments and simulations, J. Magn. Reson. 185 (2007). 159-163.

12. L. Chopin, R. Rosanske, T. Gullion, Simple improvements in spinning-speed control for MAS NMR experiments , J. Magn. Reson Series A 122, (1996) 237239.

13. Sharon E. Ashbrook, Stephen Wimperis, Rotorsynchronized acquisition of quadrupolar satellite-transition NMR spectra: practical aspects and double-quantum filtration, J. Magn. Reson. 177 (2005) 44-55.

14. Y.-H. Tsenga, Y. Moua, C.-Y. Moua, J. C.C. Chana, Double-quantum NMR spectroscopy based on finite pulse RFDR, Solid State Nuclear Magn. Reson. 27 (2005) 266270.

15. J. Heller, A. C. Kolbert, R. Larsen, M. Ernst, T. Bekker, M. Baldwin, S. B. Prusiner, A. Pines, and D. E. Wemmer, Solid-state NMR studies of the prion protein HI fragment, Protein Sci. 5 (1996) 1655-1661.

16. D.G. Cory, J.C. de Boer, W.S. Veeman, Magic angle spinning $1 \mathrm{H}$ NMR imaging of polybutadiene/polystyrene blends, Macromolecules 22 (1989) 1618-1621.

17. T. Kailath, Lectures on Wiener and Kalman Filtering, ICMS, Springer Verlag, New York 1981.

18. S. D’Arco, F. Ponci, F. D. Doty, J. Staab, Velocity and pressure measurements for microturbine control in NMR application, Instr. and Meas. Tech. Conf. 2007.

19. Wong, Kon Max, and Qu Jin, Estimation of the timevarying frequency of a signal: the Cramer-Rao bound and the application of Wigner distribution, Acoustics, Speech and Signal Processing, IEEE Transactions on 38.3 (1990) 519-536.

20. W. Kester, Converting Oscillator Phase Noise to Time Jitter, Analog Devices, MT-008 Tutorial 2008.

21. L. Baltusis, Use of audio signals for monitoring sample spinning speeds in nuclear magnetic resonance spectrometers, US Patent 5644235 (1997).

22. A. Llor and J. Virlet, Towards high-resolution NMR of more nuclei in solids: sample spinning with timedependent spinner axis angle, Chem. Phys. Lett. 152 (1988) 248-25.

23. B. F. Chmelka, K. T. Mueller, A. Pines, J. Stebbins, Y. $\mathrm{Wu}$, and J. W. Zwanziger, Oxygen-17 NMR in solids by dynamic-angle spinning and double rotation, Nature, 339 (1989) 42-43. 
24. T. Gullion, R. A . McKay, and A. Schmidt, Spin-echo, double-resonance NMR with flipped spinning (SEDORFS), J. Magn. Reson. 94 (1991) 362-369.

25. I. M. Litvak, C. A. Espinosa, R. A. Shapiro, A. N. Oldham, V. V. Duong, R. W. Martin, Pneumatic switched angle spinning NMR probe with capacitively coupled double saddle coil, J. Magn. Reson. 206 (2010) 183-189.

26. E. Mihaliuk and T. Gullion, Using heat to control the sample spinning speed in MAS NMR, J. Magn. Reson. 212 (2011) 249-253 . 
Figure 1

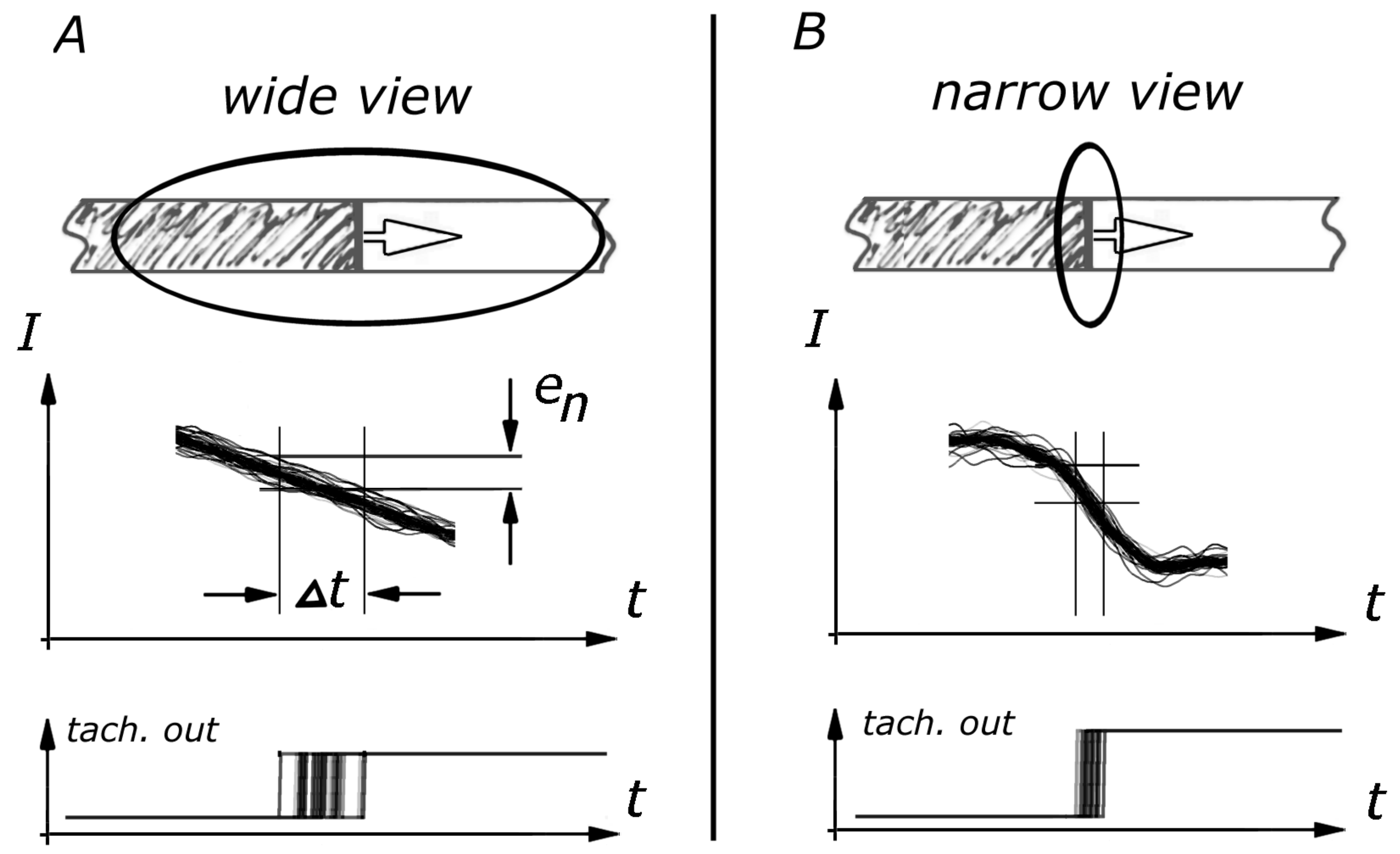


Figure 2

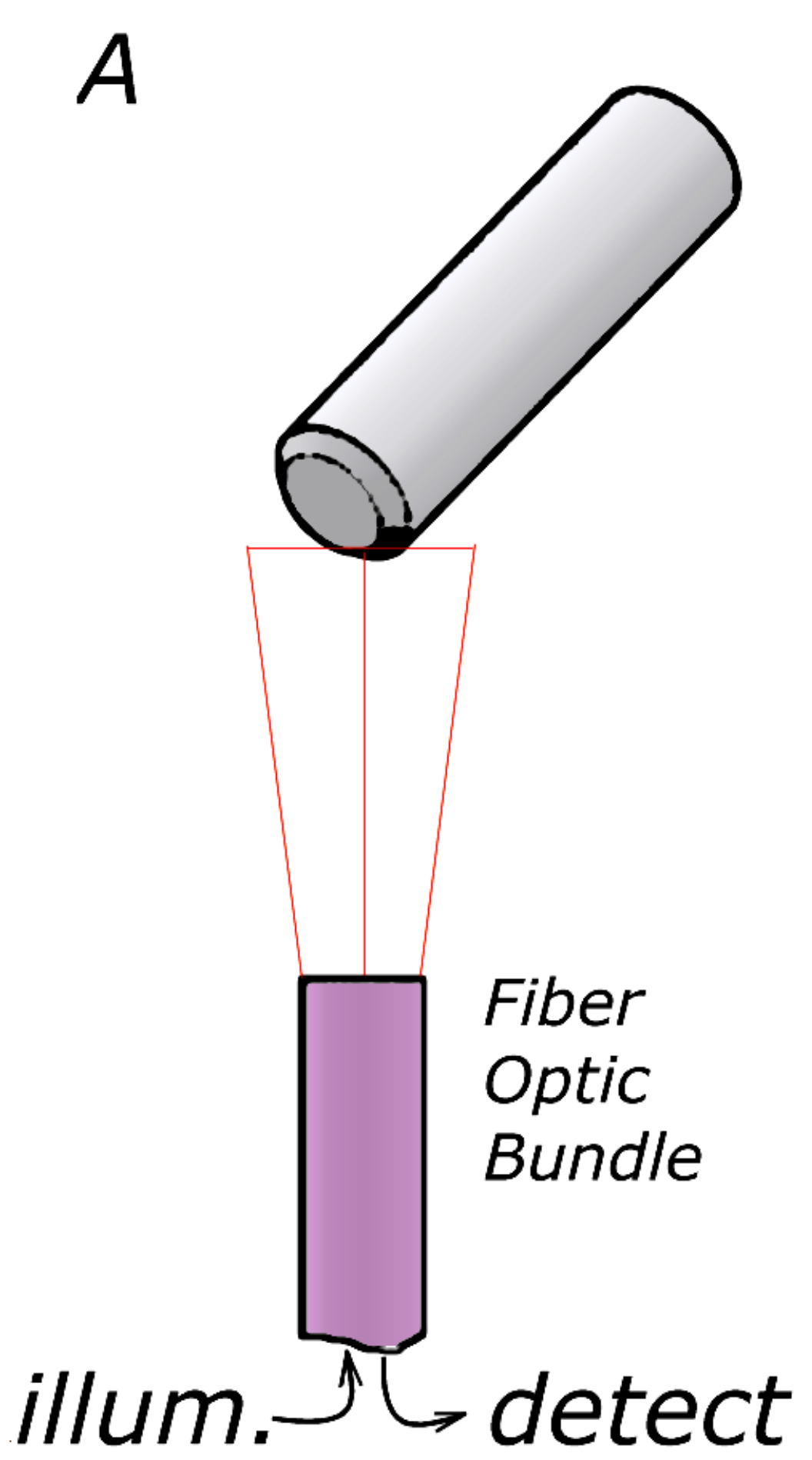

B

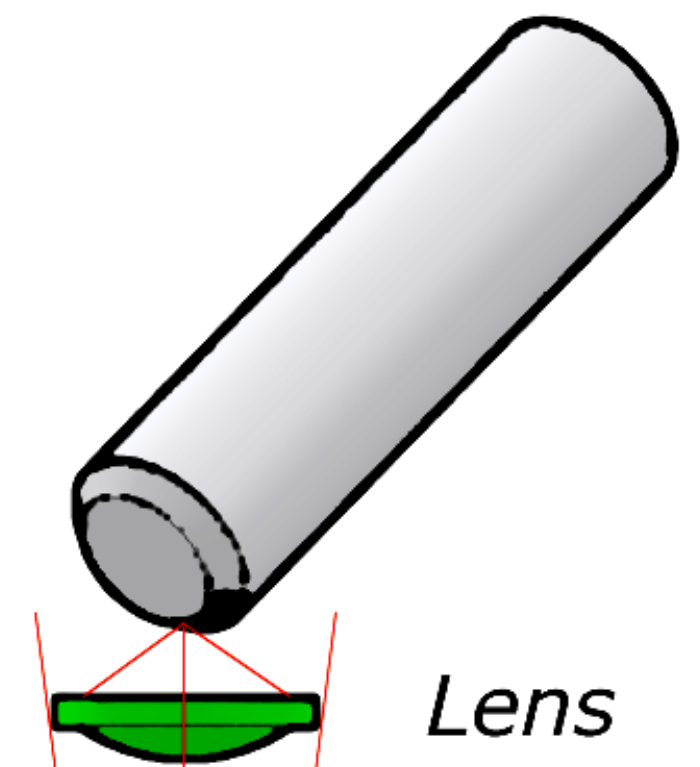

(same) 
Figure 3

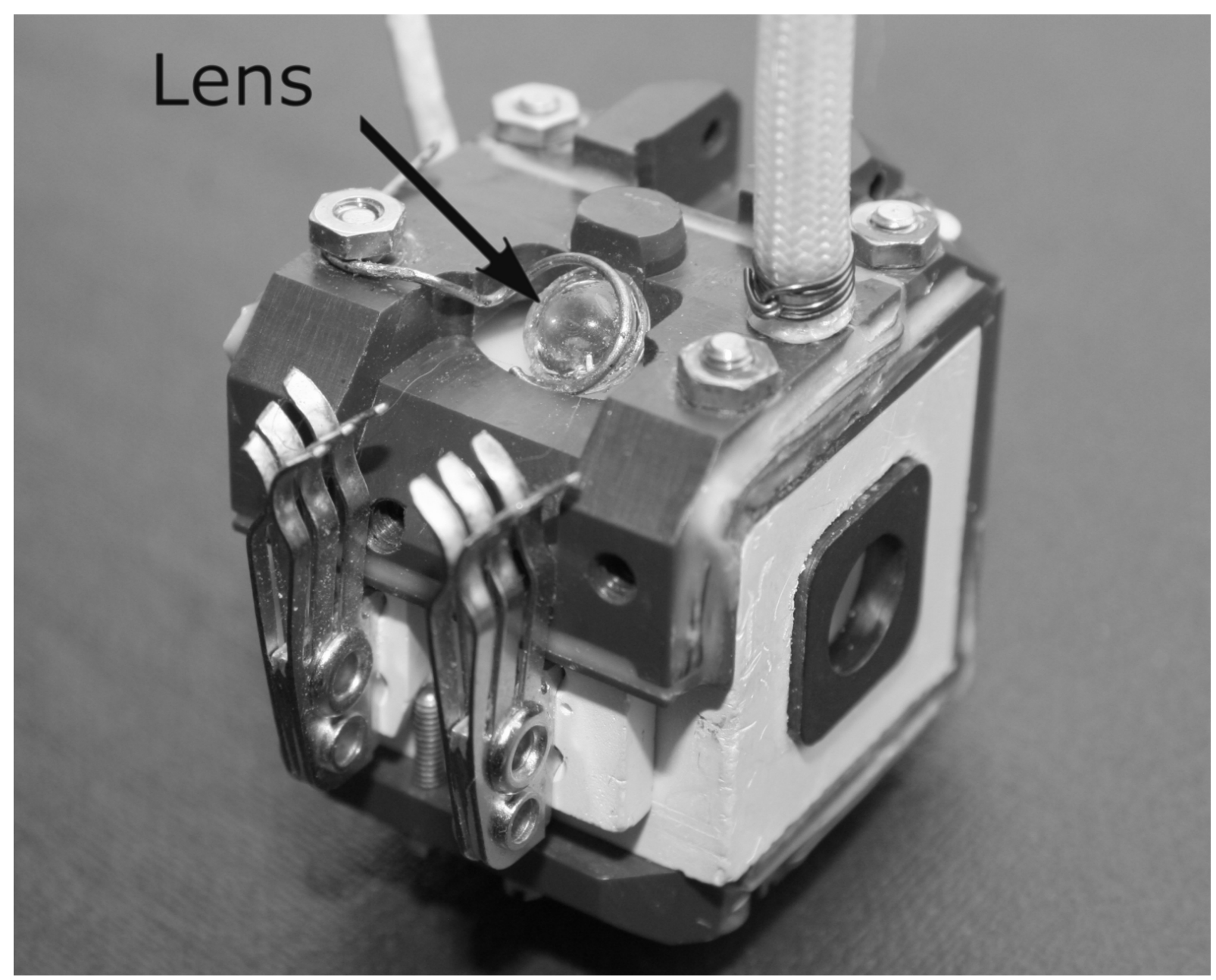


Figure 4
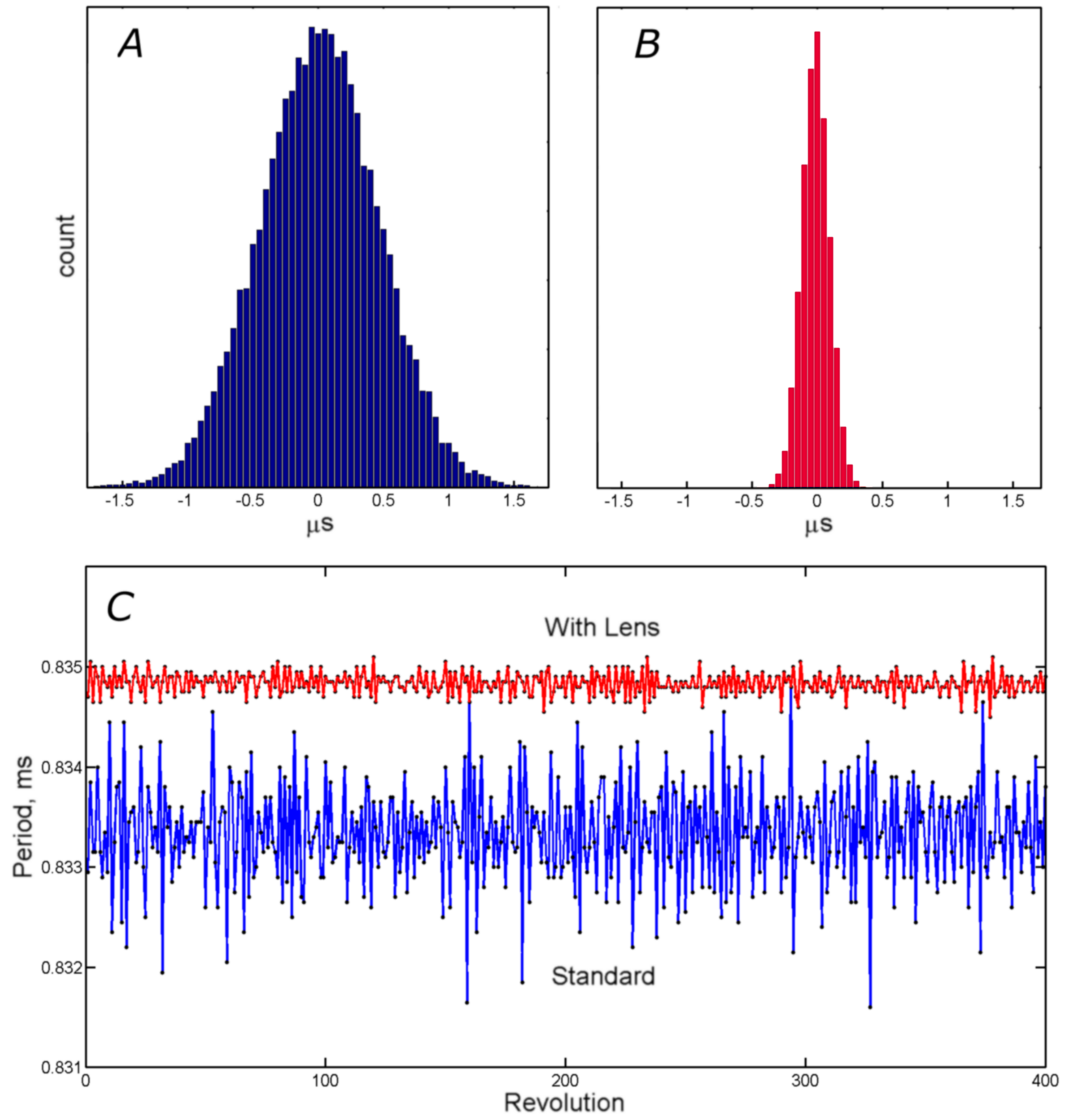
fiber optic bundle plano-convex Graphical Abstract lens

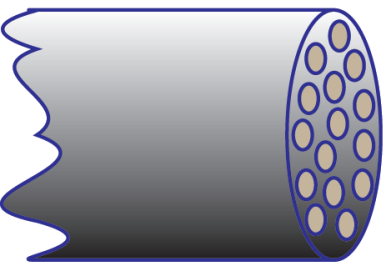

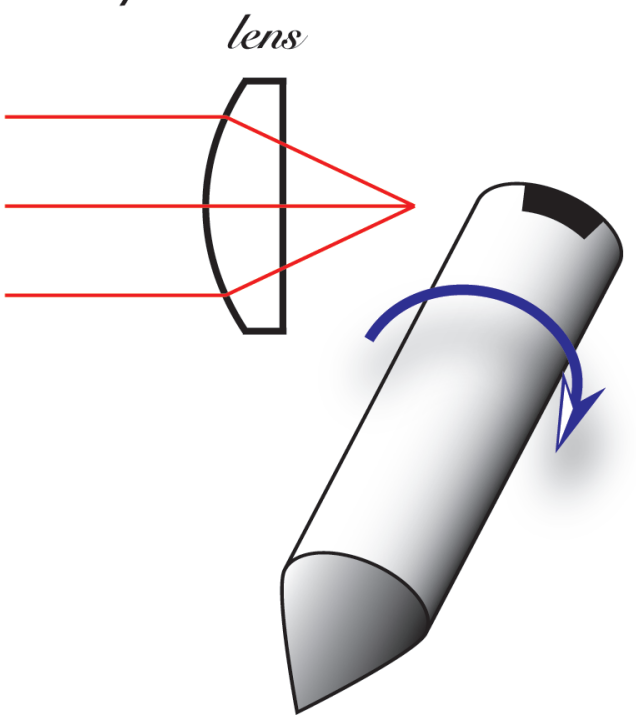

